\title{
Three Dimensional Reconstruction of Blood Vessels and Evaluation of Vascular Stenosis Based on DSA
}

\author{
Hongxing Qin ${ }^{1,2}$, Zhenyi $\mathrm{Li}^{1,2, a}$ \\ ${ }^{1}$ Chongqing Key Laboratory of Computational Intelligence Chongqing University of Posts \& \\ Telecommunications, Chongqing ,400065, China; \\ ${ }^{2}$ College of Computer Science \& Technology, Chongqing University of Posts \& Telecommunications, \\ Chongqing 400065, China; \\ alizhenyi92@163.com;
}

Keywords: DSA,CAD, three dimensional reconstructions,coronary artery,vascular stenosis.

\begin{abstract}
Cardiovascular disease is a serious threat to human health, the cause of which is mostly coronary atherosclerosis or spasm caused by vascular stenosis or obstruction. For the at the same time, digital subtraction angiography (DSA) is cardiovascular disease is the main means for modern medical diagnosis, so how to utilize the technology of Computer Aided Diagnosis (CAD) to help clinicians in order to find out and analyze the patient's symptoms, and make reasonable diagnosis have an important significance. What is put forward in the paper, a rotational DSA data of vascular 3D modeling technology, the position and angle information of medical imaging can transform the two-dimensional images of the vessels for 3D information for doctors to browse, at the same time, through the analysis on the related parameters of blood vessel diameter, provides a kind of intelligent analysis vascular stenosis degree method and clinical experiments show that by this method for inferring the vascular lesions accuracy above 95\%.
\end{abstract}

\section{Introduction}

Nowadays society, cardiovascular disease and cancer are the two leading killers of human health [1]. Currently, the clinical diagnosis methods of cardiovascular disease mainly include: digital subtraction angiography (DSA), CT tomography scanning technology and MRI imaging technology [2]. These imaging techniques can be directly or indirectly for the imaging of vascular, and provide the doctor the physiological characteristics of vascular for the diagnosis basis. Among these techniques, DSA has become the most commonly used method for diagnosis of vascular diseases because of its clear imaging and low cost [3].

However, DSA imaging technology is limited somehow, for example, use of mark fluid infusion method can make the imaging of vascular more clear, but inevitably blur the vascular diameter information, which would make difficulty to analysis vessel cavity [4]; as another example, DSA imaging is made by traditional X-ray scanning and the imaging result is two-dimensional, which is not suitable for three-dimensional diagnosis, and meanwhile rotational DSA imaging is not executed by tomography. Therefore, the traditional three-dimensional modeling technology for CT and MRI is not applicable to DSA. Although DSA equipment developed by Neusoft and PHILPS has the capability of modeling three-dimensional imaging out of the two-dimensional imaging, these methods are not universal, and these devices usually do not support the output of a three-dimensional model. The generated three-dimensional model generation is not available in the PACS system, so it is necessary to develop a general three-dimensional DSA modeling technology [5].

This paper presents a three-dimensional modeling method based on rotational DSA imaging. The shooting angle and position information is obtained from the DICOM data in DSA, real-time results of three-dimensional surface of the blood vessel wall are obtained based on the algorithm of computer vision "Structure from Motion" (SFM) [6], and finally three-dimensional vessel model with enhancement of reality is obtained using texture mapping technique. On this basis, we study the analysis technique of vascular lumen, which can calculate the target vessel diameter automatically, providing support for the clinical diagnosis of vascular stenosis. 


\section{Three-Dimensional Modeling of Blood Vessels in DSA}

\subsection{Enhancement of three-dimensional vascular.}

Because the DSA image in the region of the heart contains much non-vascular information, and these tissues will form information of different brightness degree in DSA, which causes difficulty in directly three-dimensional modeling of blood vessels from the original image. Therefore, it is necessary to extract and segment blood vessels of the DSA image.

Blood vessel shows a tubular structure in the image. Based on this feature of blood vessels, Franji, Sato etc. presented the method of identification of the tubular object using eigenvalues of Hessian matrix $[7,8]$. This algorithm can be regarded as a filter to enhance blood vessel , and can effectively enhance the blood vessels of different thickness by adding Gauss multi-scale fusion. In this paper, we carried out a lot of experiments, selected the blood vessel enhancement in a single scale, with some steps of post processing, and obtained satisfactory results.

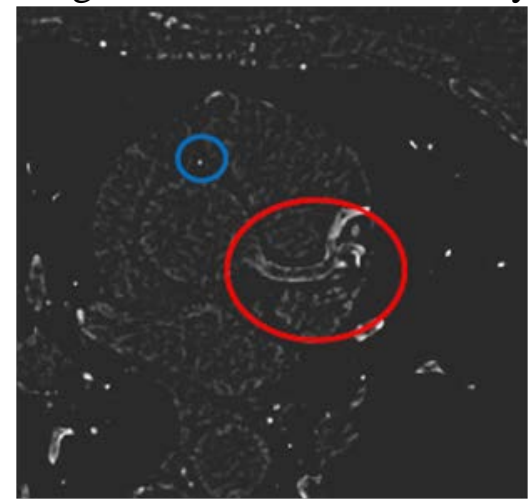

a

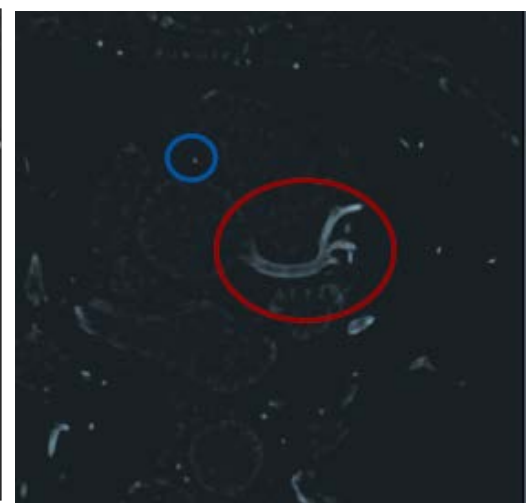

$\mathrm{b}$

Fig. 1 Vascular enhancement results based on Hessian matrix (Fig a for result before enhancement, Figure $b$ for result after enhancement).

\subsection{Calculation of Three-Dimensional Depth Informatio.}

In computer vision, assuming a three-dimensional data point in real space can be expressed as: $P_{1}=\left(X_{i}, Y_{i}, Z_{i}\right)^{T}$ and at the same time, the corresponding pixel coordinates in an image plane can be

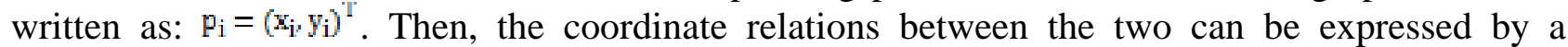
perspective model [9]

$$
A^{3 * 3}\left[\begin{array}{c}
X_{i} \\
Y_{i} \\
Z_{i}
\end{array}\right]=\left[\begin{array}{c}
x_{i} \\
y_{i} \\
K Z_{i}
\end{array}\right]
$$

In Formula $1, \mathrm{~K}$ represents a constant coefficient, $\mathrm{A}^{\mathrm{a} * \mathrm{a}}$ is the corresponding camera model (representing the X-ray machine in the DSA imaging process). The model can decompose into two matrices: $\mathrm{M}_{1}^{\mathrm{a} * \mathrm{a}}$ and $\mathrm{M}_{2}^{\mathrm{a} * \mathrm{Z}}$, as shown in Formula 2. $\mathrm{M}_{1}^{\mathrm{a} * \mathrm{a}}$ represents the internal parameters of camera lens (such as focal length and optical center position of the lens), and $\mathrm{M}_{2}^{\mathrm{a} \times \mathrm{B}}$ represents the external parameters of camera lens (such as rotational and translational information).

$$
A^{a * a}=M_{1}^{a * a} M_{2}^{a * a}=\left[\begin{array}{ccc}
a_{00}, a_{01}, & a_{02} \\
a_{10}, & a_{11}, & a_{12} \\
0, & 0, & K
\end{array}\right]
$$

We constructed a standard camera model based on formula 1 and formula 2 . If we know the internal and external parameters of camera lens, we can obtain the corresponding two-dimensional pixel $P_{i o n}$ the basis of any three-dimensional point $P_{1}$. According to the DICOM standard, the internal and external parameters related to the DSA data can be extracted directly from the 0x0008 tag group, which will not be discussed in this paper.

However, in the three-dimensional modeling process of DSA image, the coordinates of the two-dimensional pixel is known. The corresponding three-dimensional point ${ }^{P_{1}}$ need to be solved. At this time, even if the internal and external parameters of X-ray machine are known, because there are two establishable linear independent equations with three unknown parameters, and at this time, 
solving of the three-dimensional depth is an under-constrained problem which needs more constraints to be found by other methods. We introduced "Structure from Motion" theory in the field of computer vision to calculate the parameter constraints, of which the basic idea is: registration of the 2D image before the calculation of the three-dimensional depth. After registration, we can obtain the same three-dimensional point $\mathbb{P}_{1}$, according to the different internal and external parameters, projected to the two-dimensional pixel pirand pizof different images. At this point, the perspective models obtained from different angles can be united according to Formula $1 .\left[\begin{array}{ccc}x_{1}^{1} & \cdots & x_{n}^{1} \\ \vdots & \ddots & \vdots \\ x_{1}^{m} & \cdots & x_{n}^{m}\end{array}\right]=\left[\begin{array}{c}A^{1} \\ \vdots \\ A^{m}\end{array}\right]\left[\begin{array}{lll}X_{1} & \ldots & X_{n}\end{array}\right]$ $\mathrm{M}$ for $\left[\begin{array}{ccc}x_{1}^{1} & \cdots & x_{n}^{1} \\ \vdots & \ddots & \vdots \\ x_{1}^{m} & \cdots & x_{n}^{m}\end{array}\right]$, A for $\left[\begin{array}{c}A^{1} \\ \vdots \\ A^{m}\end{array}\right]$ an

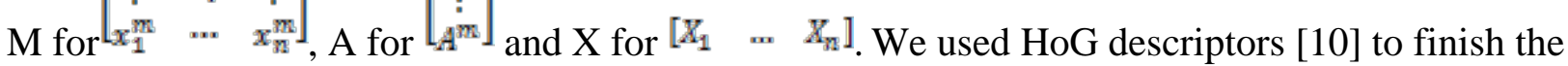
DSA image registration. In Formula 3, M represents the HoG tracking results in the DSA image sequence, $m$ represents the corresponding image index number, $n$ represents the index number of the registration in the image tracking, A represents the corresponding camera parameter matrix for $\mathrm{m}$ $\mathrm{X}$-ray camera, $\mathrm{X}$ is the three-dimensional reconstruction result based on $\mathrm{M}$ and $\mathrm{A}$, and every element $\mathrm{x}_{\mathrm{n}}^{\mathrm{m}}$ in $\mathrm{M}$ represents a registration point.

Usually in the actual "Structure from Motion" problem, as long as the data in accordance with the requirements of the matrix transformation relations described above can be obtained, data optimization algorithms (such as least square method, singular value decomposition, etc.) can be used to obtain X [11]. In actual DSA examination, in order to carry out a comprehensive diagnosis of vascular, we generally use rotating ring shot. X-ray imaging obtained at the same time contains only part of vascular structure, which makes HoG feature points in the continuous DSA images flickering, eventually leading to a lot of uncertain $\mathrm{x}_{\mathrm{n}}^{\mathrm{m}}$ in the matrix $\mathrm{M}$. At the same time, there is noise in the DSA image, and after HoG image registration, there will be a large number of false matching points, which leads to a large number of incorrect in $\mathrm{M}$. So after obtaining the initial three-dimensional depth by the theory of "Structure from Motion", it is necessary to optimize the depth of the reconstruction results.

\subsection{Optimization of Three-Dimensional Depth.}

We used light constraint algorithm to optimize the initial three-dimensional model in this paper. In the field of computer vision, light constraint algorithm is a commonly used method in optimizing the structure of the model reconstruction. Noise impact can be processed well with this method, and the internal and external parameters of the camera can be optimized by re-projection error minimization through iterative method [12].

Assuming a set of three-dimensional points in a projection model surface is $\mathrm{X}=\left[\begin{array}{ll}\mathrm{X}_{1}, & \mathrm{X}_{2,}, \mathrm{X}_{\mathrm{g}, \ldots o} \mathrm{X}_{n}\end{array}\right\}$ :

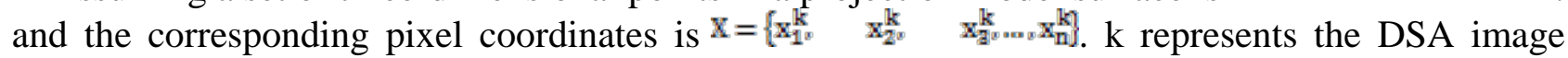
index involved in the light constraint algorithm, and n represents the number of reconstruction points. First of all, the three-dimensional point $\mathrm{P}$ in Formula 1 model is projected back to the corresponding

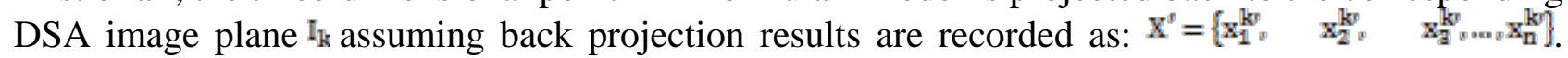
The difference value between back projection results and original DSA image pixels is the

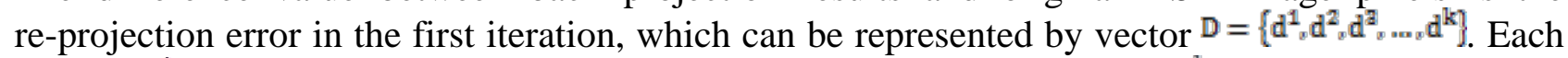
element $\mathrm{d}^{\mathrm{k}}$ in vector represents the sum of distance error between all pixels ${ }^{\mathrm{x}_{1}^{\mathrm{kj}}}$ of in the image $\mathrm{K}$ back projection and the original pixels $\mathrm{x}_{\mathrm{i}}^{\mathrm{k}}$ (as in Formula 4)

$$
\mathrm{d}^{\mathrm{k}}=\sum_{\mathrm{i}=1}^{\mathrm{n}} \frac{1}{2}\left(\mathrm{x}_{\mathrm{i}}^{\mathrm{k}}-\mathrm{x}_{\mathrm{i}}^{\mathrm{k}}\right)^{\mathrm{T}}\left(\mathrm{x}_{\mathrm{i}}^{\mathrm{k}}-\mathrm{x}_{\mathrm{i}}^{\mathrm{k}}\right)
$$

Where $\mathrm{k}$ is the image index of the current calculation, $\mathrm{i}$ is the $\mathrm{i}$ reconstruction point in the image $\mathrm{K}$. in general, Formula 4 is a function associated with some variables. We assume that these variables can be represented by a vector $\mathrm{C}^{\mathrm{B}}$, and $\mathrm{C}^{\mathrm{B}}$ is related to the splicing process and the complete camera parameters, which can be recorded as:

$$
\mathrm{C}^{\mathrm{K}}=\left\{\mathrm{P}^{\mathrm{K}}{ }_{v} \mathrm{R}^{\mathrm{K}} \mathrm{T}^{\mathrm{K}}{ }_{v} \mathrm{~K}\right\}
$$

In Formula $5, \mathrm{P}^{\mathrm{k}}$ represents the projection results of DSA image $\mathrm{k}, \mathrm{R}^{\mathrm{k}}$ and $\mathrm{T}^{\mathrm{k}}$ represent the rotation parameters and translation parameters of the X-ray camera lens of image $\mathrm{k}$, and $\mathrm{K}$ represents the internal parameters of the X-ray camera lens. Therefore, ${ }^{\mathrm{d}^{\mathrm{k}}}\left(\mathrm{C}^{\mathrm{k}}\right)$ can be regarded as a nonlinear 
function associated with the back projection error on the image $\mathrm{K}$. In order to minimize the projection

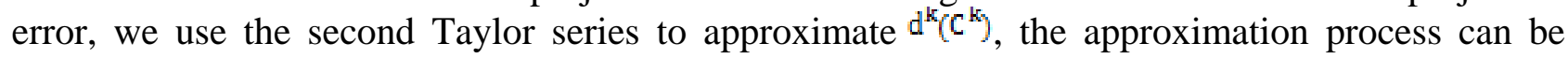
expressed as:

$$
\mathrm{J}^{\mathrm{T}} \mathrm{J} \Delta\left(\mathrm{C}^{\mathrm{k}}\right)=\mathrm{J}^{\mathrm{T}} \mathrm{d}^{\mathrm{k}}
$$

In this formula, $\mathrm{J}$ represents the Jacobian matrix, and $\Delta\left(\mathrm{c}^{\mathrm{k}}\right)$ represents the iterative optimization factor which can predict better assessment results .

Through the above process, we continuously evaluated the error of the matching points obtained from three-dimensional point and the corresponding 2D image by iterative algorithm, and adjusted the parameters to globally optimize the accuracy. However, in an actual DSA inspection, there will be tens of thousands of splice points and camera parameters, and these data make Formula 6 a high complexity problem of high dimension when calculating the global panorama. In this case, when the initial condition is poorer, light constraint algorithm may be closer to a local minimum value in the calculation, which leads to the failure of optimization. In our algorithm, we use the internal and external parameter information contained in the DICOM file to make initial selection of the original DSA image, which guarantees that our algorithm has a good initial condition.

In addition, in order to avoid the calculation results close to the local minimum, light constraint algorithm is usually designed to an increasingly progressive calculation method [13]. The main idea of the increasingly progressive light constraint algorithm is: first, using a small portion of the data as the initial input object of light constraint algorithm, and in the iterative optimization process, gradually adding subsequent data to the algorithm in the process of calculation. For example, the increasingly progressive light constraint algorithm is applied in the process of the reconstruction of three-dimensional structure from disorderly data in this study.

In this study, new image is uninterrupted inserted into the iterative process of reconstruction, and experimental results show that the algorithm can effectively avoid the local minimum problem even in dealing with massive disorder data. Some scholars have designed the input data into triples in order to avoid the emergence of the local optimal solution [14]. The experimental results show that the algorithm can improve the accuracy of three-dimensional reconstruction and the optimization of lens parameters. In this paper, we imitated the increasingly progressive calculation method. Assuming the

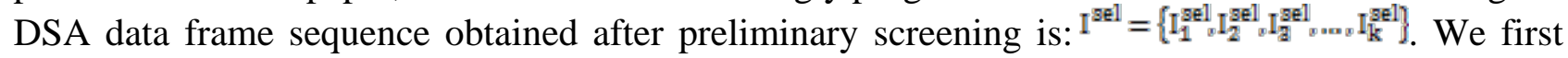
select the data set for initial light constraint from $\Delta n_{\mathrm{BA}}$, the input data set of the initial light constraint algorithm is determined by a predefined frame interval, and the selected initial subset can be recorded

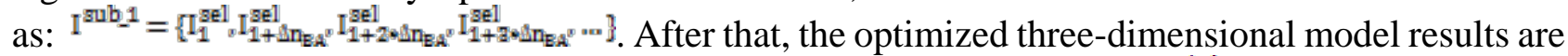
evaluated using the light constraint algorithm, based on the initial subset $\mathrm{I}^{\text {sub-1 }}$.

In the following iteration, the rest of the DSA image subsets will be selected, and recorded as:

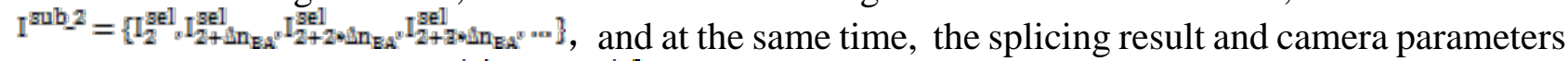

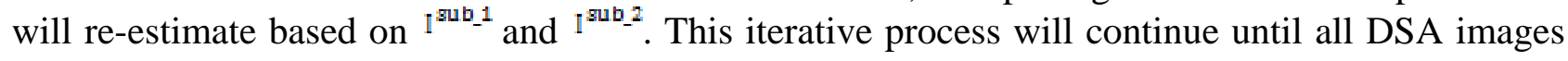
are involved in the estimation process.

\subsection{D Reconstruction Results and Accuracy.}

According to the above method, the three-dimensional model of the DSA blood vessel wall was constructed, and the accuracy of the reconstruction model was evaluated by the clinical experts of professional experience. The algorithm results show that the average accuracy of the reconstructed three-dimensional model is $97.2 \%$, which has a certain clinical value. 


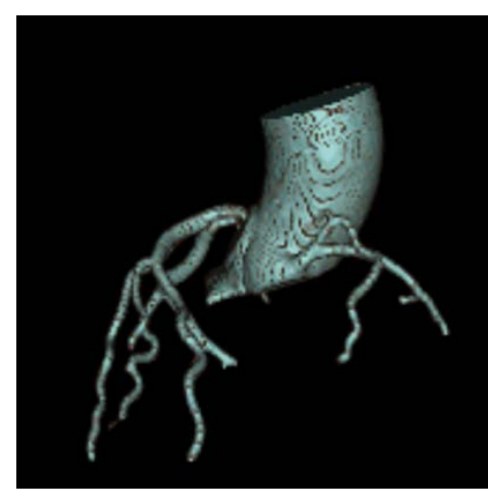

$\mathrm{a}$

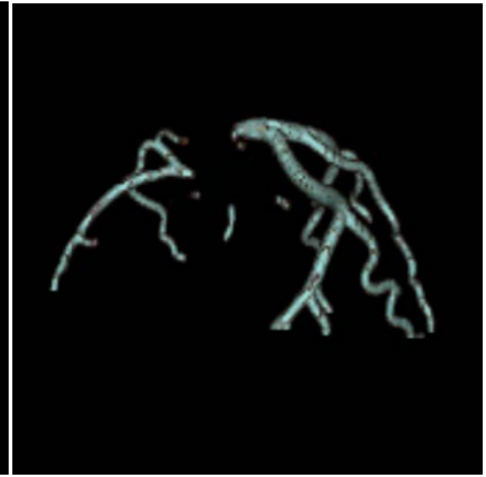

b

Fig 2 three-dimensional modeling results (Picture a for aorta and coronary artery. Picture b for coronary artery)

\section{The Calculation of Vascular Stenosis Degree}

The three-dimensional modeling method of DSA blood vessel image is introduced in detail in this paper. Three-dimensional reconstruction of the three-dimensional surface data is used to display the tree structure of coronary artery, as well as the small branches. Through careful observation, the position of the coronary artery stenosis can be located by the human eye. However, for the specific circumstances of the stenosis, such as the specific coordinates, degree of stenosis, are not quantitative results, which would not provide substantial help for the diagnosis and treatment of coronary artery disease. So we studied a method for automatic analysis of the vascular stenosis degree, which is used as the application of this paper. Our method is based on the three-dimensional modeling, radially cutting the blood vessels along the vascular extending direction to obtain the vascular section in all aspects of, and eventually getting the vascular stenosis analysis results by the statistics of the perimeter of each section.

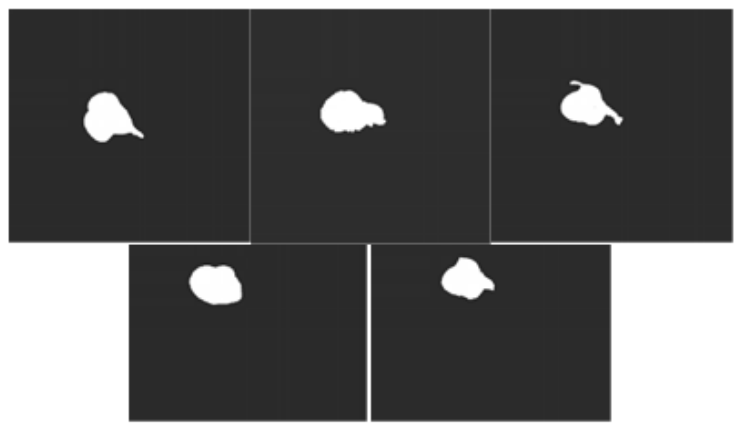

. a

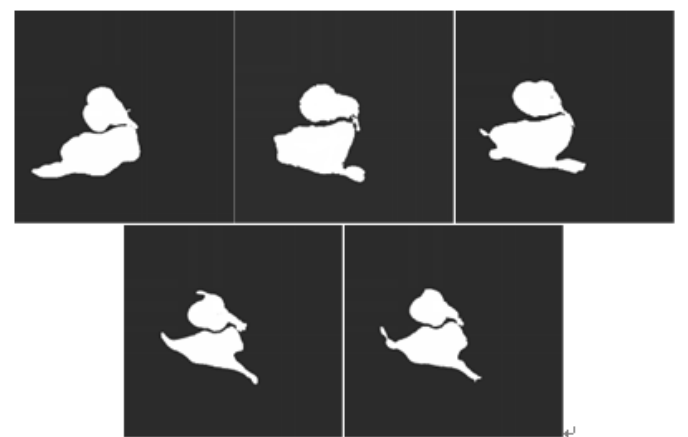

b

Fig 3 Radial Sectional View of the Vessel in DSA Three-Dimensional Model

In clinical trials, we ask the clinician to manually select the vein segments which need to analyze the stenosis degree in the reconstructed three-dimensional model, and take the clinician's diagnosis results as the gold standard to evaluate the stenosis degree using the algorithm described in this paper. The following chart is the analysis data selected by the clinician and the evaluation results of clinical trials. 


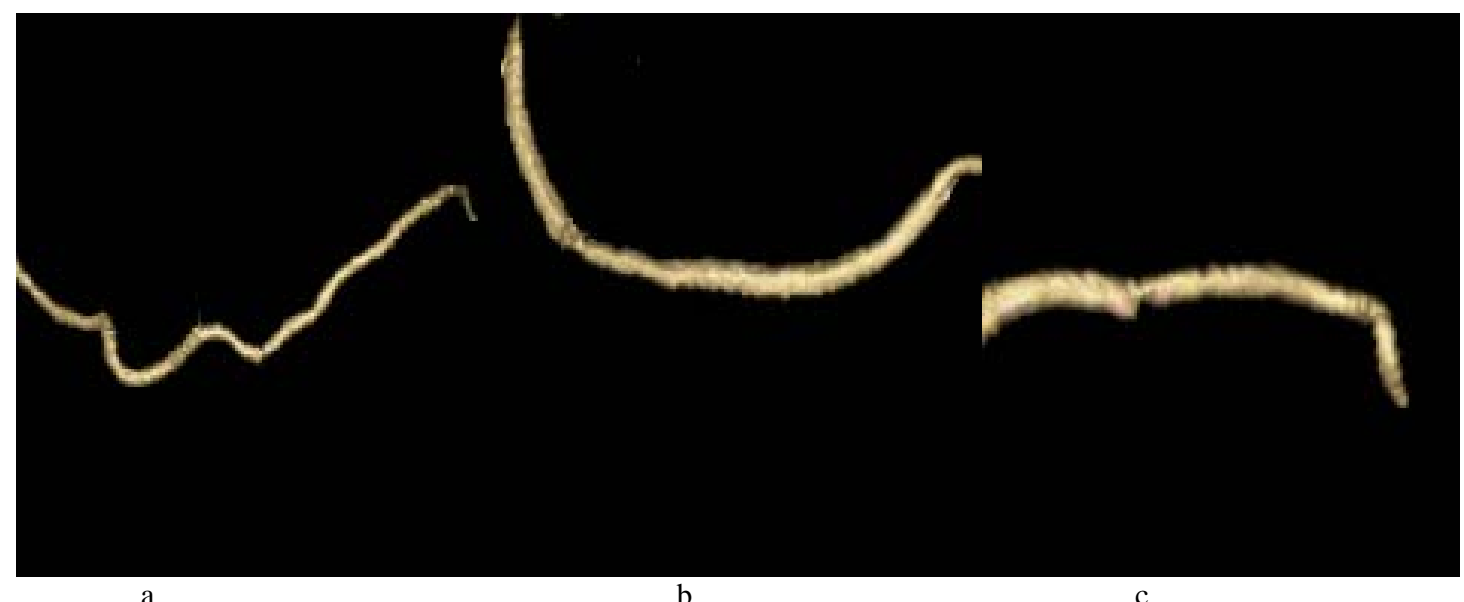

Fig 4 Three-Dimensional Model of the Target Blood Vessel
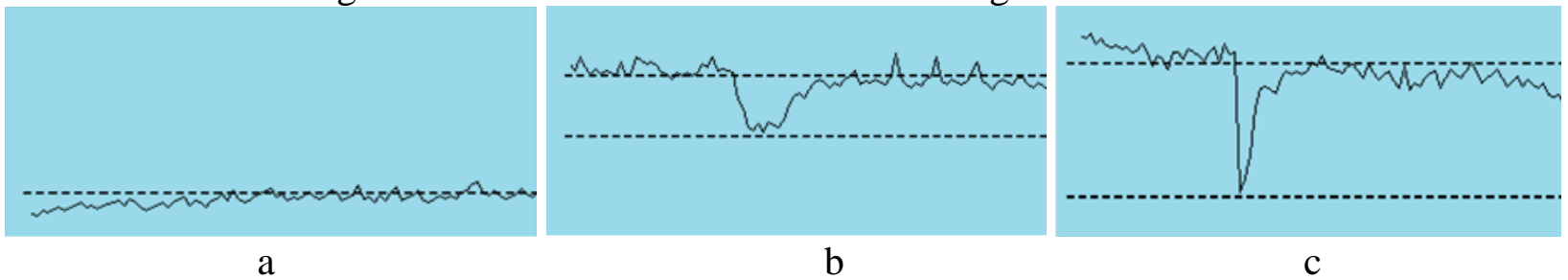

Fig 5 Graph of the measuring results of the blood vessel segment, latitudinal direction is the points found from the start point to the end, and longitudinal direction is the area size of blood vessel section of this point

Coronary artery is generally divided into 4 grade lesions according to lumen area reduction ratio: $1 \% \sim 25 \%$ for grade $1 ; 26 \% \sim 50 \%$ for grade $2 ; 51 \% \sim 75 \%$ for grade $3 ; 76 \% \sim 100$ for grade 4 . The results from Figure 5 indicate vascular lesions. In Figure 5 (b), vessels reach grade 2 lesions, (c) vessels reach grade 3 lesions, and the diagnosis results are in accordance with the diagnosis results given by the clinicianReferences.

\section{Summary}

In this paper, we study a general vascular three-dimensional modeling method founded on DSA data, which could help doctors to analyze and diagnose vascular symptoms from the three-dimensional perspective. At the same time, we present a computer aided diagnosis method of automatic analysis of stenosis degree based on the three-dimensional modeling researches. Clinical results show that the method of a certain extent can help medical diagnosis. In the future, we will do more further clinical validation and algorithm optimization.

\section{Acknowledgement}

Fund Project: National Natural Science Foundation of Youth Science Fund (61100113); Ministry of education students returning fund [2012]940; The first batch of young backbone teachers project in Chongqing City (Chongqing Education (2011) 31).

\section{References}

[1] Dayi Hu. Change idea to prevent cardiovascular disease in China[J]. Chinese Journal of Cardiology, 2008, 36(7), p.577-580.

[2] Zhuhua Zhang, Zhengyu Jin, Dongjing Li, et al. Control study of multi-slice spiral CT of coronary artery with conventional coronary angiography[J]. Journal of Clinical Radiology, 2005, 23(9),p.772-776. 
[3] Zhaoxia Zhang, Hongxiao Li, Xiaodong Chen, et al. three-dimensional segmentation of coronary artery based on the probabilistic decision and region growing[J]. Journal of Tianjin University: natural sciences and engineering technology, 2011, (5),p.454-457.

[4] Yu An. DSA cardiovascular image segmentation and visualization[D]. Beijing Jiaotong University, 2012.

[5] Gang Chen, Xuan Lv, Zhicheng Wang, et al. Lung DSA images of blood vessels skeletonization method[J]. Computer science, 2013, (5),p.274-278.

[6] Chen, P. and D. Suter, Simultaneously estimating the fundamental matrix and homographies. Robotics, IEEE Transactions on, 2009. 25(6),p.1425-1431.

[7] Frangi A F, Niessen $\mathrm{W}$ J, Vincken $\mathrm{K}$ L, et al. Multiscale vessel enhancement filtering[M]//Medical Image Computing and Computer-Assisted Interventation-MICCAI'98. Springer Berlin Heidelberg, 1998,p.130-137.

[8] Sato Y, Nakajima S, Shiraga N, et al. Three-dimensional multi-scale line filter for segmentation and visualization of curvilinear structures in medical images[J]. Medical image analysis, 1998, 2(2),p.143-168.

[9] Cleary, K. and T.M. Peters, Image-guided interventions: technology review and clinical applications. Annual review of biomedical engineering, 2010. 12, p.119-142.

[10] Zitova, B. and J. Flusser, Image registration methods: a survey. Image and vision computing, 2003. 21(11),p.977-1000.

[11] Baker, S. and I. Matthews, Lucas-kanade 20 years on: A unifying framework. International journal of computer vision, 2004. 56(3), p.221-255.

[12] Zhang, Z., et al., A robust technique for matching two uncalibrated images through the recovery of the unknown epipolar geometry. Artificial intelligence, 1995. 78(1),p.87-119.

[13] Manniesing R, Viergever M A, Niessen W J. Vessel enhancing diffusion: A scale space representation of vessel structures [J]. Medical Image Analysis, 2006, 10(6),p.815-825.

[14] National Electrical Manufacturers Association. Digital imaging and communications in medicine [J]. Document PS, 2004, 3. 